Daniel Weidner · Sigrid Weigel (Hrsg.)

Benjamin-Studien 1 

Daniel Weidner · Sigrid Weigel (Hrsg.)

Benjamin-Studien 1 
Bibliografische Information der Deutschen Nationalbibliothek

Die Deutsche Nationalbibliothek verzeichnet diese Publikation in der Deutschen Nationalbibliografie; detaillierte bibliografische Daten sind im Internet über http://dnb.d-nb.de abrufbar.

Alle Rechte, auch die des auszugsweisen Nachdrucks, der fotomechanischen Wiedergabe und der Übersetzung, vorbehalten. Dies betrifft auch die Vervielfältigung und Übertragung einzelner Textabschnitte, Zeichnungen oder Bilder durch alle Verfahren wie Speicherung und Übertragung auf Papier, Transparente, Filme, Bänder, Platten und andere Medien, soweit es nicht $\$ \$ 53$ und 54

URG ausdrücklich gestatten.

(C) 2008 Wilhelm Fink Verlag, München

(Wilhelm Fink GmbH \& Co. Verlags-KG, Jühenplatz 1, D-33098 Paderborn)

Internet: www.fink.de

Layout: Marietta Damm, Zentrum für Literatur- und Kulturforschung Berlin Einbandgestaltung: Evelyn Ziegler, München

Printed in Germany

Herstellung: Ferdinand Schöningh GmbH \& Co KG, Paderborn

ISBN 978-3-7705-4637-4 


\section{Texte und Kontexte. Zur Bedeutung des Kontextwissens für die Benjamin-Edition}

Editionen separieren Texte von den Kontexten, in denen sie bei ihrem Entstehungsprozess mit der Hand und technischen Schreibwerkzeugen unterschiedlichster Art geschrieben, als Erstdruck publiziert und von den Zeitgenossen ursprünglich rezipiert worden sind. Zugleich generieren sie, je nach ihren editorischen Prämissen, für die späteren Leser durch die von ihnen vollzogene Selektion, die Anordnung nach Textsorten oder die Anwendung anderer Strukturierungsregeln sowie durch die dabei vorgenommene Hierarchisierung, Kommentierung oder auch Nicht-Kommentierung ihrerseits neue Kontexte und konstruieren so ein bestimmtes, die Rezeption lenkendes Profil von Autor und Werk. Das ist, wie bei anderen prominenten Autoren, auch in der Wirkungsgeschichte Walter Benjamins der Fall.

Ich möchte diesem Spannungsverhältnis von Dekontextualisierung und Rekontextualisierung im Folgenden am Beispiel der sogenannten ‘kleineren` literaturkritischen Arbeiten - schon das Epitheton ist ein Beispiel für den erwähnten Selektions- und Hierarchisierungsprozess - nachgehen, also anhand der Kritiken und Rezensionen, die Benjamin seit der zweiten Hälfte der 1920er Jahre in der Frankfurter Zeitung, der Literarischen Welt und an anderer Stelle veröffentlicht hat und deren Korpus im Wesentlichen in dem 1972 publizierten dritten Band der Gesammelten Schriften publiziert worden ist. Ich werde mich dabei, in Blick auf den für diesen Beitrag zur Verfügung stehenden Raum, auf die Skizze zentraler Grundprobleme, auf pointierte Thesen und exemplarische Beispiele beschränken - in der Hoffnung, gerade durch solche Verknappung und Pointierung Impulse für eine weiterführende Diskussion der angesprochenen Probleme zu liefern. Einleitend werde ich zunächst einige grundsätzliche Fragen der Benjamin-Edition skizzieren. Im Anschluss daran werde ich die aktuelle Editionslage von Benjamins Arbeiten zur Literaturkritik im Hinblick auf die Leitfrage meines Beitrags, also auf das Spannungsverhältnis von Text(en) und Kontext(en), kritisch analysieren. Schließlich soll anhand einiger ausgewählter Rezensionen exemplarisch gezeigt werden, welche Bedeutung die in der bisherigen Editionspraxis meist ausgeblendeten Kontexte (z.B. Publikations-, Medien-, 


\section{HEINRICH KAULEN}

Wissenschaftskontexte, biografisch-soziale Kontexte, historische und politische Kontexte etc.) für ein Verständnis des Literaturkritikers Benjamin gewinnen können, sofern man ihnen editorisch die gebührende Beachtung schenkt. Dabei werden Umrisse eines neuen und anderen Benjamin-Bildes sichtbar, das sich von dem bisher verbreiteten nicht nur in Nuancen unterscheidet.

\section{Grundprobleme der Benjamin-Edition}

Editionsprojekte sehen sich im Fall Benjamins mit einer großen Herausforderung konfrontiert. Einerseits sind dessen Schriften in einer, gemessen an den Zeit- und Lebensumständen, geradezu erstaunlichen Vollständigkeit, Breite und Vielfalt archivalisch konserviert. Von den erst vor einiger Zeit aus Anlass einer Ausstellung publizierten ersten Sprachversuchen seines Sohnes Stefan ${ }^{1}$ über das 2006 veröffentlichte Adressbuch aus dem Exil ${ }^{2}$ bis zu den letzten Dokumenten aus dem Sterbezimmer in Port Bou ${ }^{3}-$ unendlich vieles ist unter den schwierigsten Bedingungen gerettet und der Nachwelt überliefert worden. Noch viel mehr ist, derzeit noch unpubliziert, in den Archiven in Berlin, Jerusalem, Paris und Moskau erhalten geblieben. Andererseits sind die meisten Texte von ihrem Verfasser, abgesehen von den wenigen Buchpublikationen und den autorisierten Zeitungsdrucken, in der Regel nicht mehr selbst in eine gültige und autorisierte Fassung gebracht worden. Vielfach blieb es bei der Absicht, das weitaus meiste jedoch ist allenfalls verstreut in Zeitschriften gedruckt, vieles blieb unvollendet oder wurde zu Lebzeiten jedenfalls nicht publiziert. Was wir heute als Benjamins Euvre identifizieren, ist - in weitaus größerem Umfang, als wir das bei anderen Autoren kennen - daher das Resultat einer posthumen Konstruktion und Rekonstruktion, die, abgesehen von der Edition von Einzelwerken wie der Berliner Kindheit um 1900 (1950), fünfzehn Jahre nach seinem Tod mit der zweibändigen Ausgabe der Schriften (1955) begonnen und mit den Gesammelten Schriften (1972-1999) und den Gesammelten Briefen (1995-2000) einen nur erst vorläufigen Abschluss gefunden hat.

Die Grundkonflikte, die aus diesem Umstand für jeden editorischen Umgang mit Benjamin resultieren, lassen sich in einigen Fragen zuspitzen: Inwiefern kommt den Schriften, die Benjamin hinterlassen hat, überhaupt der Status eines autonomen, definitiv abgeschlossenen, Werks` im Sinne einer geordneten und strukturierten Totalität von

1 Walter Benjamins Archive. Bilder, Texte und Zeichen, hg. v. Walter Benjamin Archiv, Frankfurt a.M. (Suhrkamp) 2006, S. 76-119.

2 Walter Benjamin: Das Adressbuch des Exils 1933-1940, hg. v. Christine Fischer-Defoy, Leipzig (Koehler \& Amelang) 2006.

3 Ingrid Scheurmann (Hg.): Neue Dokumente zum Tode Walter Benjamins, Bonn (AsKI e.V.) 1992. 
Texten zu, die man nach den gängigen Regeln des Wissenschaftsbetriebs bestimmten Disziplinen und Diskurspraktiken eindeutig zuordnen oder nach literaturwissenschaftlichen Gattungsgesetzen klassifizieren kann? Wie verhält sich der unterstellte Werkcharakter seiner Schriften zu dem faktischen Prozesscharakter des unablässigen Neuansetzens, Korrigierens und Wiederbeginnens, der für Benjamins Arbeit als Autor so charakteristisch ist? Dieser Werkprozess beginnt ganz konkret mit der meist handschriftlichen (oder diktierten) Niederschrift, also mit dem Schreibprozess als einem zugleich materiellen und ideellen Vorgang, und endet nur vorläufig mit der Veröffentlichung einer bestimmten Textgestalt, die bei Benjamin meist weitere - und im Prinzip niemals an ein Ende kommende - Korrekturen und Bearbeitungsschritte nach sich zieht. Diese Arbeitsweise lässt die Ergebnisse des Schreibprozesses in weiten Teilen als ein diskontinuierliches und offenes, ebenso unabgeschlossenes wie unabschließbares 'work in progress verhält sich zweitens die Geschlossenheit und Linearität der Textpräsentation, die jede Edition des Gesamtwerks - ob als Ordnung nach Werkgruppen und Textsorten oder in chronologischer Abfolge - in irgendeiner Weise herstellt, zur Fragmentarizität und Unabgeschlossenheit vieler Texte, die Benjamin hinterlassen hat, und zu seinem Bestreben, die Übergänge zwischen strikt separierten Wissensbereichen möglichst transparent und passierbar zu halten? Schließlich: wie steht die offene oder verdeckte Hierarchisierung, die Nachlasseditionen durch Selektion, Anordnung oder Kommentierung vornehmen, zur ganz und gar nicht hierarchischen Pluralität und Heterogenität der Themen, Genres und Sprachspiele, die wir im Korpus von Benjamins Texten vorfinden und die für nicht wenige Leser gerade deren spezifischen Reiz ausmachen? Es gibt zudem - und das erschwert das Problem noch mehr - auch eine Pluralität von Lesern, von denen die einen (und zwar mutmaßlich die Mehrheit) primär am Verständnis von Gedankengang und Argumentation, wiederum andere am figuralen Zeichencharakter der Schrift und an der Materialität des Schreibprozesses interessiert sind.

Ich kann und möchte auf die drei angeschnittenen Fragen keine abschließende Antwort mit einem dogmatischen Geltungsanspruch präsentieren. In den meisten Fällen muss bei der Edition eines Autors eine sachlich vertretbare, reflektierte und vor den Lesern offen gelegte Balance zwischen den genannten Polen gefunden werden. Es geht mir an dieser Stelle jedoch primär um etwas Anderes: ich möchte vor allem die Probleme ins Bewusstsein heben, die sich, unabhängig von den jeweils getroffenen editorischen Entscheidungen, bei jeder Ausgabe eines Textkorpus von der Art des Benjaminschen stellen müssen. 


\section{Anmerkungen zur Editionslage von Benjamins Arbeiten zur Literaturkritik}

Wie geht die bislang vollständigste Ausgabe von Benjamins literaturkritischen Arbeiten in den Gesammelten Schriften (GS III, erschienen 1972) mit den soeben skizzierten Problemen und Dilemmata um? Unbestritten ist, dass der von Adorno und Scholem initiierten und vor allem von Rolf Tiedemann (im Fall des dritten Bandes von Hella Tiedemann-Bartels) betreuten Edition das große Verdienst gebührt, das Benjaminsche Textkorpus zum ersten Mal umfassend gesichtet, entziffert und, ohne den Anspruch auf die Vollständigkeit einer historisch-kritischen Edition zu erheben, in seinen maßgeblichen Teilen dem kulturellen Gedächtnis allererst wiedergeschenkt zu haben. Aus diesem Grund möchte ich den alten, von Helmut Heißenbüttel, Hannah Arendt und der Studentenbewegung bereits Mitte der 1960er Jahre aus Anlass der ersten Briefausgabe (1966) eröffneten `Benjamin-War über die Frankfurter Edition hier nicht mit neuen Pauschalurteilen fortsetzen, sondern in erster Linie einige weiterführende Perspektiven erschließen. Denn in der historischen Retrospektive können wir heute schärfer die Prämissen erkennen, die dieser Rekonstruktion zugrunde liegen, und die Akzentsetzungen und Blickverengungen, die damit unweigerlich verbunden sind. Ich resümiere in der gebotenen Kürze einige wesentliche Probleme:

1. Die Ausgabe setzt eine Hierarchisierung zwischen (kunstphilosophischen) Hauptwerken und (literaturkritischen, literarischen, feuilletonistischen) Nebenwerken fort, die im Ansatz bereits in der frühen Auswahl von 1955 erkennbar war, nun aber zum Organisations- und Gliederungsprinzip einer voluminösen, mehrbändigen Gesamtausgabe erhoben wird. Obwohl Benjamin sich selbst immer wieder, etwa in seinem Brief an Siegfried Kracauer vom 5.6.1927, entschieden zur »kleinen Form«bekannt hat (GB III, 263), wird alles, was wohl zu dieser Rubrik zu zählen wäre, gleichsam unter >Vermischtes` abgelegt und in den dritten bzw. vierten (später auch in den sechsten) Band der Gesamtausgabe hineingezwängt, sofern es bis heute überhaupt publiziert worden ist (etliche Fragmente sind aufgrund dieser Selektionsprinzipien gänzlich unveröffentlicht geblieben). Die Inhaltsverzeichnisse der entsprechenden Bände gestalten sich entsprechend wenig einladend. Man muss nur in die Teile der Gesamtausgabe hineinschauen, welche die (vermeintlichen) Hauptwerke und die, wie suggeriert wird, bedeutungsvolleren Essays und Abhandlungen enthalten, um den Unterschied zu ermessen. Hatte es in der Einbahnstraße aber im Einspruch auch gegen solche Praktiken nicht gerade geheißen, dass »alle entscheidenden Schläge [...] mit der linken Hand geführt werden« (GS IV.1, 89)? 
2. Die Hierarchisierung setzt sich fort in der Beziehung zwischen den im Hauptteil abgedruckten, rabgeschlossenen Texten und den dazu gehörigen Alternativfassungen, Entwürfen, Fragmenten und Paralipomena, die - sei es vollzählig oder selektiv - in einen mehr oder weniger komplexen und selbst für geübte Leser unübersichtlichen, teilweise mit Materialien zur Entstehungs- und Wirkungsgeschichte überfrachteten und von Wertungen und Interpretationen keineswegs immer ganz freien Anhang abgedrängt werden. Der Werkcharakter wird auf diese Weise gegenüber dem Prozesscharakter eindeutig favorisiert.

3. Die Selektion, Anordnung und Kommentierung der Texte stehen unverkennbar im Zeichen eines bestimmten Deutungskanons, der - wie in vielen vergleichbaren Fällen (man denke beispielsweise an die Geschichte der Kafka- oder der Bachmann-Editionen) - bei der ersten Werkausgabe von der Sichtweise der Weggefährten und Freunde, hier der von Scholem und insbesondere von Adorno, inspiriert worden ist. Adorno hat nie geleugnet, dass Benjamin aus seiner Sicht "gerade als Philosoph wiederhergestellt werden mußte. ${ }^{4}$ Noch deutlicher wird Adorno in einem Brief an Hannah Arendt vom 2. Mai 1967, in dem zugleich eine Ahnung von den Schwierigkeiten mitschwingt, tatsächlich das philosophische Zentrum von Benjamins weit verzweigten Denkperspektiven und Arbeitshorizonten auszumachen:

Für mich ist axiomatisch, wodurch Benjamins Bedeutung in meiner eigenen geistigen Existenz umschrieben wird: das Wesen seines Denkens als eines philosophischen. Nie habe ich unter einem anderen Gesichtspunkt seine Sachen sehen können, und es will mir scheinen, daß sie dadurch allein ihr ganzes Gewicht finden. Wie sehr das freilich von aller traditionellen Auffassung von Philosophie sich entfernt, ist mir gegenwärtig, und darüber hinaus: daß Benjamin es einem nicht leicht gemacht hat, eben diese Ansicht von ihm festzuhalten. ${ }^{5}$

Der Fokus der Gesammelten Schriften richtet sich aufgrund der hier explizit formulierten Prämissen primär auf den Philosophen Walter Benjamin und seinen Beitrag zur Kunst-, Sprach- und Geschichtstheorie der Moderne. Dies ist zwar eine legitime, für die Reaktualisierung Benjamins im kulturellen Gedächtnis möglicherweise auch zeitweilig unverzichtbare Sichtweise, die aber, sofern sie sich zum Deutungsmonopol verfestigt, wichtige andere Facetten des vielseitigen Intellektuellen, Forschers und philosophierenden Lite-

4 Theodor W. Adorno an Helmut Heißenbüttel, 14.4.1967, in: Schreibheft. Zeitschrift für Literatur 67 (September 2006), S. 186.

5 Arendt und Benjamin. Texte, Briefe, Dokumente, hg. v. Detlev Schöttker/Erdmut Wizisla, Frankfurt a.M. (suhrkamp taschenbuch wissenschaft, 1795) 2006, S. 181. 


\section{HEINRICH KAULEN}

raten Benjamin ausblendet oder zumindest marginalisiert und daher weder den aktuellen Standards der Editionswissenschaft noch den heutigen Einsichten der Benjaminforschung gerecht werden kann. Sichtbar wird die Propagierung eines bestimmten, bis heute nachwirkenden Benjaminbildes etwa in einer Kommentierung, welche primär die Affinitäten zu ausgewählten Einflussgrößen nachzeichnet und durch diese Kontextualisierung den Autor in einem großflächigen Diskursnetz positioniert, das in der Regel von denselben dominanten Instanzen besetzt wird, zumeist in Gestalt der intellektuellen Dreierkonstellation Adorno, Scholem und - allerdings lediglich als Randfigur - Brecht. Von den konkreten sozialen, publizistischen und intellektuellen Kontexten, in denen sich Benjamin damals bewegt hat und zu dem viele weitere - heute teils bekannte, teils vergessene - Namen gehören, erfährt man bei diesen Rekonstruktionen meist reichlich wenig, und der Leser sollte sich hüten, die skizzierte Konstruktion intellektueller Einflusssphären mit diesem tatsächlichen Lebensumfeld zu verwechseln.

In welchem Maße die Edition der Rezensionen in den Gesammelten Schriften spezifischen Leitbildern folgt, zeigt sich aber auch an der vorgenommenen Textselektion. Texte wie »Ein deutsches Institut freier Forschung«(1938; GS III, 518-526), die gemeinsam mit Adorno verfassten »Vorschläge für den Besprechungsteil der ,Zeitschrift für Sozialforschung` (GS III, 601f.) oder der Essay über »Probleme der Sprachsoziologie«(1935, GS III, 452-480) haben unter den Rezensionen, schaut man näher hin, eigentlich nichts zu suchen. Ihre Aufnahme verdankt sich anscheinend weniger sachlichen Kriterien als den unterstellten intellektuellen Konvergenzen. Auch die zeitliche Selektion der Texte folgt offenkundig den oben erwähnten Prämissen: Bis auf drei unbedeutende Ausnahmen (eine von 1912, zwei von 1924), sind nur solche Kritiken aufgenommen worden, die nach 1926 geschrieben worden sind. Suggeriert wird damit eine Zäsur in Benjamins Biographie, die mit dem Scheitern des Frankfurter Habilitationsplans zusammenhängt, das Benjamin von seiner eigentlichen Bestimmung, der akademischen und philosophischen Forschung, abgehalten und in der Folge zur Übernahme minderer publizistischer Auftragsarbeiten genötigt habe.

Gekoppelt ist dieses Klischee mit dem zur Identifikation einladenden Stereotyp des melancholischen und scheiternden Linksintellektuellen, der, gegenüber seinen eigenen Auftraggebern selbst angeblich nahezu machtlos, an der fatalen Übermacht der Verhältnisse zerbrochen sei. Dass Benjamins Tätigkeit als Literaturkritiker weder erst 1926 beginnt noch auf eine der genannten Formeln gebracht werden kann, wird aus dem Bewusstsein der Leser verdrängt. 


\section{TEXTE UND KONTEXTE}

4. Die beschriebene Kontextualisierung Benjamins im weiteren Umfeld der Kritischen Theorie steht nur scheinbar in Widerspruch zu der Beobachtung, dass gerade im Apparatteil des hier exemplarisch herangezogenen Bands durch Verzicht auf Kommentierungen zum institutionellen und sozialen Umfeld des Kritikers zugleich massiv mit Entkontextualisierungsstrategien gearbeitet wird. Nur selten erfährt man etwas über sozio-biografische, kulturwissenschaftliche, wissenschaftshistorische oder politische Zusammenhänge, über die publizistischen und medialen Rahmenbedingungen oder über die Strategien Benjamins bei der Platzierung seiner Arbeiten. In welcher sachlichen oder persönlichen Beziehung dieser zu den von ihm behandelten Autoren und Gegenständen steht, kann der Leser meist nur erahnen. Dies ist vor allem erstaunlich, wenn man an die geradezu ausufernde Kommentarpraxis in etlichen anderen Bänden der Gesamtausgabe denkt. Aber auch der Verzicht auf Erläuterungen ist in diesem Fall eine spezifische Art von Kommentierung. Implizit ist damit nämlich unterstellt, dass die genannten Kontextbezüge und mithin der »Traditionsraum«(GS III, 97), in dem die präsentierten Texte stehen, für deren Verständnis unwichtig seien, weil es sich ja ohnehin in der Regel um ungeliebte Auftragsarbeiten zum Zweck des puren Broterwerbs handele. „Wissenschaftliche Nacktkultur»hat Benjamin dieses bewusste Ausklammern zentraler Kontexte an anderer Stelle einmal polemisch genannt (GS III, 97) - in seiner Rezension zu Eva Fiesels Romantikbuch (1928), die selbst übrigens keineswegs unproblematisch ist, zumal wenn man sie unter genderspezifischen Aspekten und im Blick auf wissenschaftsinterne Ausgrenzungsprozesse kontextualisiert. 


\section{Editorische Probleme am Beispiel ausgewählter Rezensionen}

Im Gegensatz zu der soeben skizzierten Praxis möchte im Folgenden zeigen, welche Relevanz das Wissen um bestimmte Kontexte für das adäquate Verständnis von Benjamins Tätigkeit als Rezensent und Literaturkritiker haben kann. Ich beziehe mich dabei vorrangig auf (1) soziobiografisches, (2) literaturhistorisches und (3) publizistisches Kontextwissen und ziehe bewusst solche Beispiele heran, die noch nicht zu den kanonisierten Teilen von Benjamins Euvre gehören.

1. 1930 rezensiert Benjamin für die Literarische Welt das kleine Büchlein von Gabriele Eckehard Das deutsche Buch im Zeitalter des Barock (GS III, 236f.). ${ }^{6}$ Der Kommentar der Gesamtausgabe verweigert dazu jegliche weitere Informationen. Nicht einmal die wahre Identität der Verfasserin, die in Koschs Literaturlexikon rasch zu ermitteln ist, wird aufgelöst. Dabei kannte Benjamin sie persönlich gut: hinter dem Pseudonym Gabriele Eckehard verbirgt sich nämlich Lucy Helene Domke, die Ehefrau des jüdischen Rechtsanwalts Martin Domke (1892-1980), einem engen Freund, dem in späteren Jahren die Überführung wichtiger Nachlassteile in die USA zu verdanken ist. Es ist anzunehmen, dass Benjamin, der damals Domkes Lichtenberg-Sammlung in dessen Auftrag katalogisierte, auch die Barocksammlung von Domkes Frau, die den Anlass für das besprochene Büchlein geliefert hat, aus eigener Anschauung gekannt hat. Eine weitere Pointe besteht darin, dass die Verfasserin ihrerseits in ihrem Literaturverzeichnis auf das von der Öffentlichkeit damals kaum beachtete Trauerspielbuch verweist. Das sind zweifellos philologische Petitessen. Für das Verständnis von Benjamins Intention bei der Publikation seiner Rezension und erst recht für die Einschätzung des Umstands, dass der Kritiker, wie schon Klaus Garber gezeigt hat, die Dürftigkeit der kleinen Abhandlung mit »höflichem Schweigen« überging, ${ }^{7}$ sind solche `Nebensächlichkeiten ‘ aber keineswegs unwichtig.

Auch in anderen Fällen kann das Wissen um biografische Kontexte durchaus hilfreich sein, um zu begreifen, dass Benjamin die Besprechung eines Buches keineswegs nur aus Zufall oder finanzieller Not übernommen hat. Tom Seidmann-Freud etwa, von der er 1930/31, scheinbar ohne erkennbaren Anlass, zwei Spielfibeln bespricht (GS III, 267-272, 311-314) kannte der Literaturkritiker ebenfalls persönlich. Sie war eine Nichte Sigmund Freuds, lebte 1919 bis 1921 in München bei derselben Vermieterin wie Gershom Scholem und war entfernt mit dessen zweiter Ehefrau verwandt. In Berlin machte sie sich später

\footnotetext{
Vgl. das Faksimile in: Walter Benjamins Archive (Anm. 1), S. 28.

Klaus Garber: Walter Benjamin als Briefschreiber und Kritiker, München (W. Fink) 2005, S. 219. Auch Garber durchschaut bereits das Pseudonym ,Gabriele Eckehard.
} 
einen Namen als bedeutendste Bilderbuchkünstlerin der Weimarer Zeit und fiel dabei dem passionierten Kinderbuchexperten Benjamin auf. Dieser bewunderte die jüdische Bohèmienne aber schon seit seinem Besuch in München im Jahr 1921. ${ }^{8}$ Auch in seinen Rundfunkvorträgen weist er an einer Stelle lobend auf sie hin (GS VII.1, 107).

Auf eine lange zurückliegende Bekanntschaft geht auch die Besprechung zu David Baumgardts Werk über Franz von Baader zurück (1931, GS III, 304-308). Baumgardt hatte sich 1924 in Berlin mit der von Benjamin sieben Jahre später besprochenen Arbeit habilitiert und damit das geschafft, was diesem bekanntlich verwehrt geblieben war. Er war schon ein Bekannter aus gemeinsamen Studienjahren, in denen Benjamin seinen jüdischen Kommilitonen einmal zum Vortrag vor der Freien Studentenschaft eingeladen hatte (GB I, 214f.). Später verbrachte Baumgardt ein Gastsemester bei Scholem in Jerusalem.

Diese drei zufällig ausgewählten Beispiele, die sich ohne jede Mühe vermehren ließen, zeigen, dass Benjamins Tätigkeit als Rezensent an Stellen, an denen man es kaum vermutet, vielfach auf einem dichten Netzwerk von Kontakten und persönlichen Beziehungen beruht. Das Bild des isolierten Außenseiters und scheiternden Melancholikers greift zu kurz und geht oftmals an den Fakten vorbei. Nur die allerwenigsten Rezensionen sind tatsächlich bloße Auftragsarbeiten und verdanken sich ausschließlich ökonomischen Zwängen. Oftmals, de facto wohl in der Mehrzahl der Fälle, war Benjamin, wie wir heute wissen, an der Titelauswahl aktiv beteiligt. In der Regel lässt sich ein sachlicher und nicht selten, wie gezeigt, auch ein soziobiografischer Bezug zu seiner Person herstellen. Um das zu erkennen, darf man sein Werk freilich nicht länger entkontextualisieren oder nur bestimmten dominanten Diskursfeldern zuordnen.

2. Ich wähle bewusst wiederum ein Beispiel, das eher zu den verdeckten und leicht übersehenen Arbeiten Benjamins gehört: die Rezension zu Ssofja Fedortschenko [Sofija Fedorčenko]: Der Russe redet. Aufzeichnungen nach dem Stenogramm aus dem Jahr 1926 (GS III, 49). Benjamin schreibt darüber am 5.4.1926 an Scholem (GB III, 134-136), das Buch bringe »ohne Anmerkungen, Daten noch Namen Sätze aus Unterhaltungen russischer Soldaten, wie eine Samariterin, die an der Front war, sie von Fall zu Fall aufgezeichnet hat. Es ist vielleicht, wahrscheinlich, das aufrichtigste und positivste Buch, welches der Krieg hervorgebracht hat. (GB III, 135). . Was Benjamin verschweigt, der von den bisherigen Benjaminausgaben allein gelassene Leser aber wissen muss, ist, dass das vermeintlich

8 Walter Benjamin/Gershom Scholem: Briefwechsel 1933-1940, hg. v. Gershom Scholem, Frankfurt a.M. (Suhrkamp) 1980, S. 35. Vgl. Gershom Scholem: Von Berlin nach Jerusalem. Jugenderinnerungen, Frankfurt a.M. 1977 (Bibliothek Suhrkamp, 555), S. $158 f$.

9 Vgl. dazu auch den Brief an S. Kracauer vom 5.11.1926 (GB III, S. 212). 


\section{HEINRICH KAULEN}

dokumentarische Werk in Wahrheit das literarische Werk einer jungen Autorin ist, die das Geschehene weniger dokumentiert als fiktive Soldatengespräche narrativ arrangiert und konstruiert hat. In den kulturpolitischen Debatten nach der Russischen Oktoberrevolution löste das Buch in Russland gerade wegen seines Anspruchs auf dokumentarische Authentizität heftige Kontroversen aus. Während Avantgardisten wie Alexander Blok das formale Experiment verteidigten, griffen die Verfechter eines ästhetischen Konservatismus das Konzept scharf an. Die Rezension berührt sich zudem nicht nur mit der Diskussion um den Kriegsroman in der Weimarer Republik, die den Literaturkritiker Benjamin zu dieser Zeit beschäftigt hat (vgl. GS VI, 165f.; entstanden um 1929/30), sondern vor allem mit den im Zeichen der Neuen Sachlichkeit auch in Deutschland geführten Diskussionen um die Ästhetik der Dokumentar- und Reportageliteratur, die von Benjamin eigentlich bekämpft und abgelehnt wird (vgl. GS VI, 180). Ohne Kenntnis dieses Kontexts bleibt dem Leser der Zugang zu dieser kleinen Buchbesprechung verschlossen. Benjamins Arbeit als Kritiker ist auch kulturell in der Regel nicht isoliert, sondern hat an wichtigen kulturpolitischen Strömungen, Tendenzen und Debatten teil.

3. Das gilt, unter veränderten Vorzeichen, auch für den letzten Text, auf den ich im Rahmen meines Beitrags eingehen möchte. 1931 publiziert Benjamin im Literaturblatt der Frankfurter Zeitung seine Rezension eines schweizerischen Kräuterbüchleins. ${ }^{10}$ Die Besprechung wäre nicht weiter der Rede wert, nähme Benjamin nicht diesen abseitigen und etwas merkwürdigen Gegenstand - auf eine für seine intellektuelle Physiognomie charakteristische Weise - zum Anlass für weit reichende Erörterungen zur Wirkungs- und Erfolgsgeschichte von Büchern, zur Funktion der Literaturkritik, zum Verhältnis von populärem und gelehrtem Wissen und zu einer Reihe anderer Probleme grundsätzlicher Art. Unter den Benjamin-Kennern genießt die kleine Rezension aus diesem Grund seit langem eine besondere Wertschätzung. Was aber auch die Experten aufgrund der Dekontextualisierungsstrategien der Gesammelten Schriften, zumindest beim Umgang mit Benjamins `kleineren` Feuilletonbeiträgen und Rezensionen, meist nicht wissen, ist, dass der Titel Wie erklären sich große Bucherfolge? anscheinend gar nicht von Benjamin selber stammt. Es handelt sich vielmehr um den Obertitel einer Artikelserie im Literaturblatt der Frankfurter Zeitung, in der von März bis Juni 1931 Kritiker wie Friedrich Burschell, Efraim Frisch und Erich Franzen zu Wort kamen. Ihre Beiträge dienten einer Bestandsaufnahme der zeitgenössischen Massenkultur und zielten darauf ab, Modeautoren der Weimarer Republik wie Remarque, Zweig oder Frank Thieß in Blick auf den Habitus

10 Wie erklären sich große Bucherfolge? "Chrut und Uchrut»- ein schweizerisches Kräuterbuch (1931; GS III, S. 294-300). 
ihres bürgerlichen Lesepublikums ideologiekritisch zu dechiffrieren. Gerahmt wurde die Serie durch zwei Beiträge von Siegfried Kracauer, von denen der erste die Intention der Artikelfolge umreißt, während der zweite, ohne auf Benjamins Besprechung näher einzugehen, eine Zwischenbilanz der Ergebnisse zieht. Die Absicht der Serie, heißt es in der Frankfurter Zeitung (Literaturblatt) vom 1.3.1931, bestehe darin, »einige Gründe zu ermitteln, aus denen es manchen Büchern gelingt, große Lesermassen zu erobern. Ein Hauptaugenmerk möchten wir bei dieser Veranstaltung auf solche Erfolgsbücher richten, die auch und vornehmlich zu den literarisch ungebildeten Schichten der Bevölkerung sprechen. « ${ }^{11}$ Dieser Intention ist, gerade in ihren programmatischen Ausführungen, offensichtlich auch Benjamins Rezension gewidmet. Allerdings nimmt diese, nicht nur im Blick auf den späten Veröffentlichungstermin, eine Sonderstellung in der Serie ein. Der Grund hat wiederum mit Benjamins spezifischem Denkansatz zu tun: während die übrigen Kritiker die Bestsellerqualitäten eines Werks ideologiekritisch auf dessen Fundierung in bürgerlichen Ideologien und Rezeptionsmustern zurückführen, Popularität mithin als Massenbetrug entlarven wollen, verfolgt Benjamin einen anders akzentuierten, differenzierteren Begriff von Popularität, der Breitenwirkung nicht einfach mit Affirmation identifiziert und Ungelehrtheit nicht schlicht mit Unwissenheit und Dummheit gleichsetzt. Das spezifische Profil der konzeptionellen Überlegungen, die er in diesem Zusammenhang gleichsam nebenbei - eben »mit der linken Hand «- vorstellt, erschließt sich freilich nur dann, wenn man sie nicht länger als isoliertes Produkt eines Außenseiters betrachtet, sondern in ihren tatsächlichen Kommunikations- und Publikationskontext re-integriert. Dazu brauchen die Benjaminleser ein zureichendes und zuverlässiges Kontextwissen, das ihnen Forschung wie Editionsvorhaben zur Verfügung zu stellen haben.

\section{Ausblick: Benjamin als Kritiker und Rezensent}

Abschließend sollen, wiederum thesenhaft-pointiert, einige der Konsequenzen angedeutet werden, die sich für das verbreitete Bild des Literaturkritikers Benjamin ergeben werden, wenn man ihn, wie gefordert, präziser und differenzierter kontextualisiert, als dies bislang der Fall gewesen ist.

Zunächst: Benjamin ist kein bloßer Auftragsrezensent gewesen, der alles übernehmen musste, was gleichsam als Brosamen von den Tischen der Redaktionen abfiel. Bei ca. 80

11 Siegfried Kracauer: Schriften, hg. v. Inka Mülder-Bach, Bd. 5.2: Aufsätze 1927-1931, Frankfurt a.M. (Suhrkamp) 1990, S. 294. Vgl. Literaturblatt der Frankfurter Zeitung, 64 Jg., Nr. 9, S. 7 (1.3.1931). 
bis $90 \%$ seiner Rezensionen war er an der Auswahl der Titel aktiv beteiligt, ${ }^{12}$ oder sie kamen den eigenen Vorlieben und Arbeitsbereichen eo ipso entgegen. Seine Praxis als Kritiker folgt, mindestens für die Zeit von 1926 bis Anfang1933 und teilweise auch darüber hinaus, erstaunlich konsequent und geradlinig bestimmten Strategien und steht fast immer in einem - mal offenen, mal verdeckten - Zusammenhang mit den Themen und Leitmotiven seines übrigen Werks. Sie ist also, anders als man lange Zeit angenommen hat, weder von subjektivistischer Willkür noch von bloßen Zufällen und äußeren Zwängen diktiert. Auf diese Weise vermochte der Kritiker bis zur völligen Gleichschaltung der deutschen Presse durch die Nationalsozialisten eine relativ stabile und erstaunlich breite Produktionspraxis zu behaupten, zu der noch weitere regelmäßige Einnahmen, vor allem aus den kontinuierlichen Rundfunkarbeiten, hinzukamen. Benjamin war zudem zeit seines Lebens sozial in mannigfache Kommunikationszusammenhänge integriert und stand in einem relativ dichten Netzwerk von Kontakten, Freundschaften und Beziehungen unterschiedlichster Art, die weit über die in der Wirkungsgeschichte bis heute dominante intellektuelle Figuration Adorno - Scholem - Brecht hinausreichen. Viele dieser Beziehungen sind inzwischen durch die neue sechsbändige Briefausgabe hinreichend dokumentiert, was noch nicht heißt, dass sie auch von der Forschung bereits in angemessener Weise wahrgenommen, geschweige denn wissenschaftlich untersucht worden wären. Speziell bei der kleinräumigen Topographie des Intellektuellen Walter Benjamin jenseits der großen Diskurslinien besteht noch reichlicher Forschungsbedarf.

Auch das Bild des melancholischen und scheiternden Intellektuellen, das sich zum Teil auf einschlägige Selbststilisierungsversuche des Autors, vor allem aus der Zeit nach 1933, berufen kann, ist zumindest für die Zeit der Weimarer Republik partiell zu korrigieren. Benjamins Arbeit als Feuilletonist, Kritiker und Rezensent ist bis 1933 keineswegs nur von Misserfolgen begleitet gewesen, vielmehr konnte der Publizist Benjamin, gemessen an seinen Möglichkeiten, eine Zeitlang durchaus mit gewissem Geschick und Erfolg als "»Stratege im Literaturkampf« (GS IV.1, 108) operieren. Er kannte sich in den publizistischen Kontexten und in den zeitgenössischen Medienensembles, speziell im Journalismus und im sich allmählich etablierenden Rundfunk, gut aus und beherrschte, wie etliche Beispiele zeigen, auch bereits die Technik der kommerziellen Mehrfachverwertung und des Medienwechsels, insbesondere im Blick auf den Transfer zwischen den alten Printmedien und den neu aufkommenden Massenmedien. Ein Vergleich der Rundfunkarbeiten

12 Dies zeigt auch die mit dem Gattungsbegriff Rezensionen überschriebene Liste mit möglichen Buchtiteln zur Besprechung, die der Kritiker in seinem Schwarzen Lederheft sorgfältig geführt und gleichsam systematisch rabgearbeitet hat; sie hat sich im Nachlass erhalten (Walter Benjamin Archiv, Berlin, Ms. 673/41r). 


\section{TEXTE UND KONTEXTE}

mit den parallel entstandenen Zeitungsbeiträgen würde diese These im Detail erhärten. Gewiss wäre es fatal, das, wie wir gesehen haben, problematische Leitbild des heroisch scheiternden Intellektuellen gegen das heute modische, aber darum vielleicht noch fragwürdigere Leitbild des spielerischen, souverän operierenden Multimedia-Akteurs auszutauschen, und das liegt auch gar nicht in meiner Absicht. Ich wollte lediglich an einigen kleineren Texten demonstrieren, wie wenig wir in vielen Fällen bis heute über die konkreten Entstehungs- und Wirkungszusammenhänge von Benjamins Schaffen tatsächlich wissen. Sie freizulegen, wird dazu beitragen, einige neue und überraschende Perspektiven auf dessen heterogenes Werk zu eröffnen. 